\title{
Stat3 Related Gene Proteins are Key Regulators in Chronic Inflammation to Lung Adenicarcinoma Transition \\ Peng Qu*
}

Vascular Biology Section, Mouse Cancer Genetics Programe, NCl/Frederick, Bldg. 560, Room 12-34, 1050 Boyles ST, Frederick, MD 21702 , USA

In the past year, we developed CCSP-rtTA/ (tetO) (7)-MMP12 bitransgenic lung cancer model to study MMP12 function in the process of inflammation-triggered lung inflammation and cancer. In this model, MMP12-Flag fusion protein overexpression and its increased enzymatic activity were observed in the lung in an inducible manner, which led to inflammatory cell infiltration and increased epithelial growth. Spontaneous emphysema and bronchioalveolar adenocarcinoma were found through pathological analysis. During this process, ELISA results demonstrated that the concentration of some cytokins was increased in bronchioalveolar lavage fluid, which activated the oncogenic signal transducer and activator of transcription 3 (Stat3) in alveolar type II epithelial cells. Real-time PCR and western blotting results demonstrate that expression of Stat 3 downstream was significantly increased in the lung. MMP12 upregulation was highly associated with patients with COPD and lung cancer.

A myeloid-specific c-fms-rtTA/(TetO) $)_{7}$-CMV-MMP12 bitransgenic mouse model was created. In this bitransgenic system, MMP12 overexpression elevated frequencies and numbers of Common Myeloid Progenitor (CMP) and Granulocyte/Macrophage Progenitor (GMP) populations, and decreased the frequency and number of the Megakaryocyte/Erythrocyte Progenitor (MEP) population in the bone marrow, through 9-color flow cytometric analysis. One specific CD11b (+)/ Gr-1(+) Myeloid Derived Suppressor Cells (MDSCs) was found to in- creased in multiple organs. Both in vitro and in vivo studies showed an immunosuppressive function on T-cell proliferation and function by MDSCs. Regulatory T cells (Tregs) were increased. In the lung, the concentration of IL-6 was increased, which aberrantly activated oncogenic Stat 3 and increased expression of Stat 3 downstream genes in epithelial tumor progenitor cells. Spontaneous emphysema and lung adenocarcinoma were sequentially developed. BM chimeras confirmed that the MMP12-induced myeloid cell autonomous defect led to abnormal myelopoiesis, immune suppression, and lung adenocarcinoma.

In those two mouse model, we found that MMP12 overexpression on myeloid cells or lung epithelial cells can induce chronic inflammation in lung (the numbers of MDSCs and regulatory T cells were increased), and lead to lung cancer. We first indicate that MMP12 may become novel gene, which can induce lung cancer.

In 2014, many questions remain to be elucidated, and are listed here:

- Are stat3 and downstrean gene regarded as markers of lung COPD or Lung cancer?

- In human cancer, the phenotypic characterization of MDSCs needs to be further developed.

- How do we enhance MDSC-based therapies in cancer patients?

*Corresponding author: Peng Qu, Vascular Biology Section, Mouse Cancer Genetics Programe, NCl/Frederick, Bldg. 560, Room 12-34, 1050 Boyles ST, Frederick, MD 21702, USA, Tel: 301-846-5692; E-mail: qup@mail.nih.gov

Received January 23, 2014; Accepted January 28, 2014; Published February 02,2014

Citation: Qu P (2014) Stat3 Related Gene Proteins are Key Regulators in Chronic Inflammation to Lung Adenicarcinoma Transition. J Blood Disorders Transf 5: e111. doi: 10.4172/2155-9864.1000e111

Copyright: @ 2014 Qu P. This is an open-access article distributed under the terms of the Creative Commons Attribution License, which permits unrestricted use, distribution, and reproduction in any medium, provided the original author and source are credited. 